EPJ Web of Conferences 101, 06009 (2015)

DOI: $10.1051 /$ epjconf/ 201510106009

(C) Owned by the authors, published by EDP Sciences, 2015

\title{
Seven-Period Asteroseismic Fit of KIC 8626021
}

\author{
Agnès Bischoff-Kim ${ }^{1, \text { a }}$, Roy H. Østensen ${ }^{2}$, J.J. Hermes ${ }^{3}$, and Judith L. Provencal ${ }^{4}$ \\ 1 Penn State Worthington Scranton, Dunmore, PA 18512, USA \\ 2 Institute of Astronomy, KU Leuven, Celestijnenlaan 200D, 3001 Heverlee, Belgium \\ 3 Department of Physics, The University of Warwick, Coventry CV4 7AL, UK \\ 4 Department of Physics and Astronomy, University of Delaware, Newark DE 19716, USA
}

\begin{abstract}
We present a new, better-constrained asteroseismic analysis of the heliumatmosphere (DB) white dwarf discovered in the field of view of the original Kepler mission. Observations obtained over the course of two years yield at least seven independent modes, two more than were found in the discovery paper for the object. With several triplets and doublets, we are able to fix the $\ell$ and $m$ identification of several modes before performing the fitting, greatly reducing the number of assumptions we must make about mode identification. We find a very thin helium layer for this relative hot DB, which adds evidence to the hypothesis that helium diffuses outward during DB cooling.
\end{abstract}

\section{Introduction}

We present results of the asteroseismic study of the only helium atmosphere pulsating white dwarf discovered in the original Kepler field, KIC 8626021. Seven quarters of consecutive data on the object allowed us to resolve 7 independent modes, including 3 triplets.

\section{Fourier Transform}

In Fig. 1, we show the fourier transform of 7 consecutive quarters of data. Setting a conservative detection limit of $5 \sigma$, we find 7 independent modes in the light curve, that we used in an asteroseismic fit of the object. From the triplet structure of $f_{1}, f_{2}$ and $f_{3}$, we learn that these modes are $\ell=1$ modes and we derive a rotation period for the star of $1.8 \pm 0.4$ days.

\section{Asteroseismic fitting}

We use a forward modeling approach to asteroseismology, whereby we assume an internal chemical structure for the star, calculate static models for that composition, and compare the periods of the model with the periods observed in the star. We are looking for the best match between the two.

To compute the models, we use the White Dwarf Evolution Code (WDEC). The WDEC is described in more detail in [1] and references therein. The period-by-period fit and mode identifications are listed in table 1 . The fitness function $\sigma_{\mathrm{RMS}}=\sqrt{\sum_{1}^{n_{\mathrm{obs}}}\left(P_{\text {model }}-P_{\mathrm{obs}}\right)^{2} / n_{\mathrm{obs}}}$ for the model periods listed in table 1 is $0.362 \mathrm{~s}$. Our best fit model has a temperature of $29,650 \mathrm{~K}$, a mass of $0.550 M_{\odot}$, a helium/carbon envelope mass of $10^{-3.10}$, the outer $10^{-7.90}$ of which is pure helium, and a central oxygen abundance of 0.55 . The edge of the homeogeneous $\mathrm{C} / \mathrm{O}$ core is located at $M_{r}=0.22 M_{*}$. Full details on the modeling and asteroseismic fitting results for KIC 8626021 were published in [1].

a e-mail: axk55@psu.edu

This is an Open Access article distributed under the terms of the Creative Commons Attribution License 4.0, which permits unrestricted use, distribution, and reproduction in any medium, provided the original work is properly cited. 


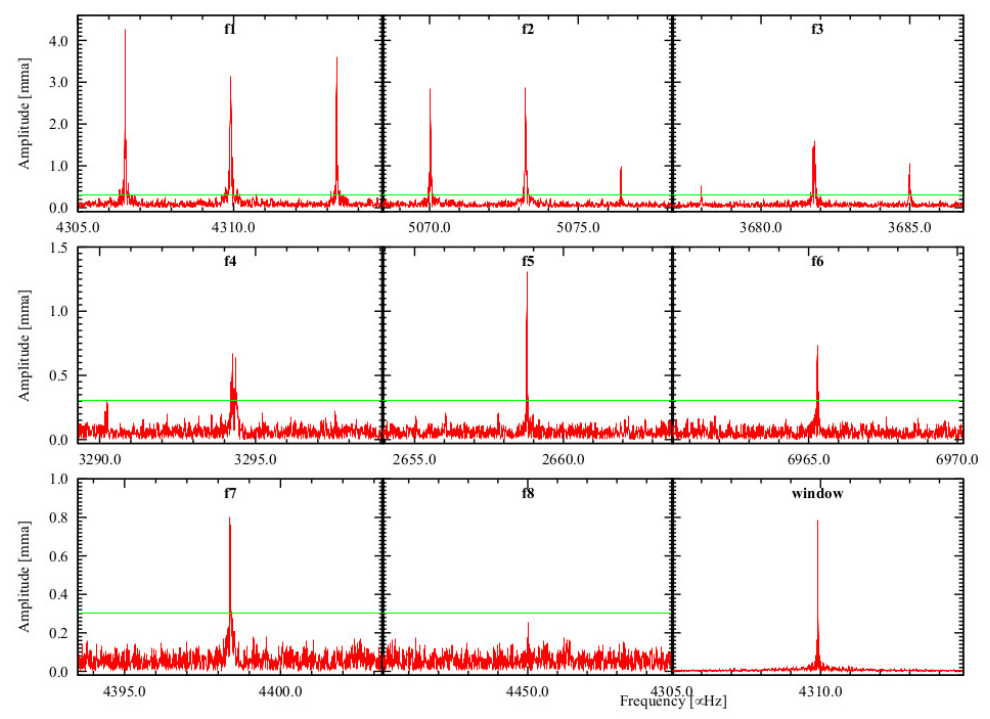

Fig. 1. Fourier peaks of the eight highest-amplitude modes, Q10 through Q17 data. The green horizontal line denotes the detection threshold set at the 5- $\sigma$ limit of $0.3 \mathrm{mma}$.

Table 1. Period list with fits

\begin{tabular}{lllllll}
\hline Frequency $[\mu \mathrm{Hz}]$ & Amplitude $[\mathrm{mma}]$ & $P_{\text {obs }}$ & $P_{\text {model }}$ & $\ell$ & $k$ & $m$ \\
\hline 6981.3 & 0.38 & 143.24 & 142.68 & 2 & 4 & 0 \\
5076.435 & 0.99 & 196.99 & & 1 & 3 & +1 \\
5073.235 & 2.87 & 197.11 & 197.60 & 1 & 3 & 0 \\
5070.03 & 2.84 & 197.24 & & 1 & 3 & -1 \\
4398.369 & 0.80 & 227.36 & 226.94 & 2 & 8 & 0 \\
4313.369 & 3.61 & 231.84 & & 1 & 4 & +1 \\
4309.919 & 3.15 & 232.02 & 231.88 & 1 & 4 & 0 \\
4306.520 & 4.29 & 232.21 & & 1 & 4 & -1 \\
3684.966 & 1.07 & 271.37 & & 1 & 5 & +1 \\
3681.800 & 1.61 & 271.61 & 271.52 & 1 & 5 & 0 \\
3677.996 & 0.54 & 271.89 & & 1 & 5 & -1 \\
3294.381 & 0.67 & 303.55 & 303.58 & 1 & 6 & 0 \\
3290.0 & 0.30 & 303.95 & & 1 & 6 & -1 \\
2658.769 & 1.31 & 376.11 & 376.51 & 1 & 8 & 0 \\
\hline
\end{tabular}

\section{Conclusions}

Our best fit model parameters are consistent with what we expect from stellar evolution calculations and from spectroscopy [2]. In particular, KIC 8626021 is both the hottest known DBV and the one fitted with the thinnest helium layer models. This is consistent with the outward diffusion of helium as DBs cool. It would be worth checking this trend quantitatively, using the other DBVs we have or can fit similarly to KIC 8626021.

\section{References}

1. A. Bischoff-Kim, R.H. Østensen, J.J. Hermes, J.L. Provencal, ApJ, 794, (2014) 39

2. D. Koester, Astronomical Society of the Pacific Conference Series 493, (2015) 129 